\title{
Ciclos solares em dados de precipitação pluviométrica registrados no Rio Grande do Sul (RS)
}

Cruz, M. F. ${ }^{*}$, UNIPAMPA; Caprara, B. B., UNIPAMPA; Herdies, G. R., UNIPAMPA; Frigo, E., UNIPAMPA.

Copyright 2018, SBGf - Sociedade Brasileira de Geofísica

Este texto foi preparado para a apresentação no VIII Simpósio Brasileiro de Geofísica, Salinópolis, 18 a 20 de setembro de 2018. Seu conteúdo foi revisado pelo Comitê Técnico do VIII SimBGf, mas não necessariamente representa a opinião da SBGf ou Técnico do VIII SimBGf, mas não necessariamente representa a opinião da SBGt ou propósitos comerciais sem prévia autorização da SBGf.

\section{Resumo}

Este trabalho tem como objetivo investigar as periodicidades presentes em dados do total anual de precipitação pluviométrica registrados em quatro estações meteorológicas localizadas no estado do Rio Grande do Sul entre os anos de 1912 e 2017. O método de análise espectral utilizado foi a Análise por Regressão Iterativa de Séries Temporais. Os resultados matemáticos obtidos indicam que o fenômeno El Niño Oscilação Sul e os ciclos solares de Schwabe e Hale podem estar relacionados com a modulação da variabilidade das chuvas na região sul do Brasil.

\section{Abstract}

The goal of this work is to investigate the presence of periodic variations in data of the total annual rainfall level, recorded in four weather stations located in the state of Rio Grande do Sul for the period between 1912 and 2017. The spectral analysis method used was the time series iterative regression analysis. The mathematical results indicate that the El Niño - Southern Oscillation phenomenon and the Schwabe and Hale solar cycles may be related to the modulation of the rainfall variability in the investigated region.

\section{Introdução}

A quantidade e a distribuição de precipitação pluviométrica ao longo do tempo são alguns dos fatores que mais influenciam na produtividade das atividades de agricultura e pecuária. Estas atividades, por sua vez, são muito importantes para a economia do estado do Rio Grande do Sul (RS). Neste cenário, o entendimento dos fenômenos que contribuem para a variabilidade da precipitação representa uma importante contribuição da ciência para o desenvolvimento da sociedade.

No último século, o território do estado do Rio Grande do Sul se encontra na região que é afetada pela Anomalia Magnética do Atlântico Sul (AMAS), a qual é caracterizada pelos menores valores de intensidade total do campo geomagnético na superfície da Terra. $O$ centro desta anomalia atravessou o território da região sul do Brasil nas últimas décadas. Resultados provenientes de análise espectral em séries de dados meteorológicos tem indicado que a AMAS exerce um papel indireto na modulação climática na região sul do Brasil, atuando de forma a intensificar efeitos relacionados com a atividade solar e os raios cósmicos galácticos (Echer et al., 2008; Rampelotto et al., 2012; Frigo, 2013).
Neste trabalho, é utilizado um método clássico de análise espectral visando identificar as variações periódicas em dados do total anual de precipitação pluviométrica, registrados em quatro estações meteorológicas instaladas no RS, cobrindo o período entre 1912 e 2017.

\section{Dados e Método de Análise}

Os dados utilizados neste trabalho foram digitalizados a RCG partir dos registros das estações arquivados no Instituto Nacional de Meteorologia (INMET). Os registros utilizados consistem dos relatórios mensais de cada estação meteorológica. Neste trabalho foram utilizados dados das estações Iraí (IRA), Passo Fundo (PFU), Santa Maria (SMA) e Bagé (BAG). Estas estações estão localizadas em diferentes latitudes no RS e são distantes da costa. A localização de cada estação está apresentada na Figura 1.

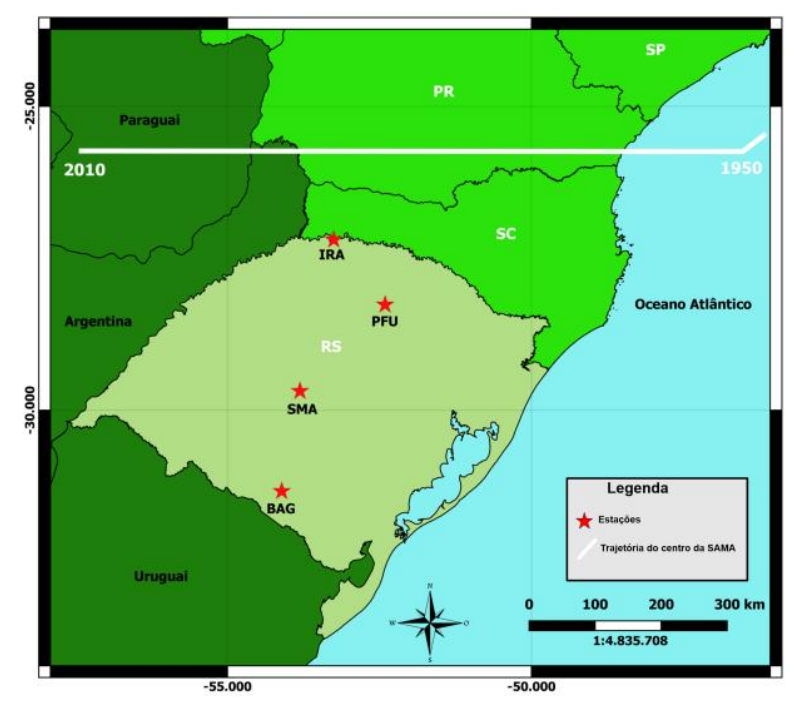

Figura 1 - Mapa de localização das estações utilizadas. A linha branca representa a trajetória aproximada do centro da AMAS.

A partir dos totais mensais de precipitação de cada estação, foram calculados os totais anuais. As séries temporais de IRA, PFU, SMA e BAG estão apresentadas na Figura 2. Com o objetivo de identificar as variações periódicas presentes em cada uma das séries temporais foi utilizado um método de análise espectral clássica. $O$ método utilizado foi a Análise por Regressão Iterativa de Séries Temporais (ARIST). 

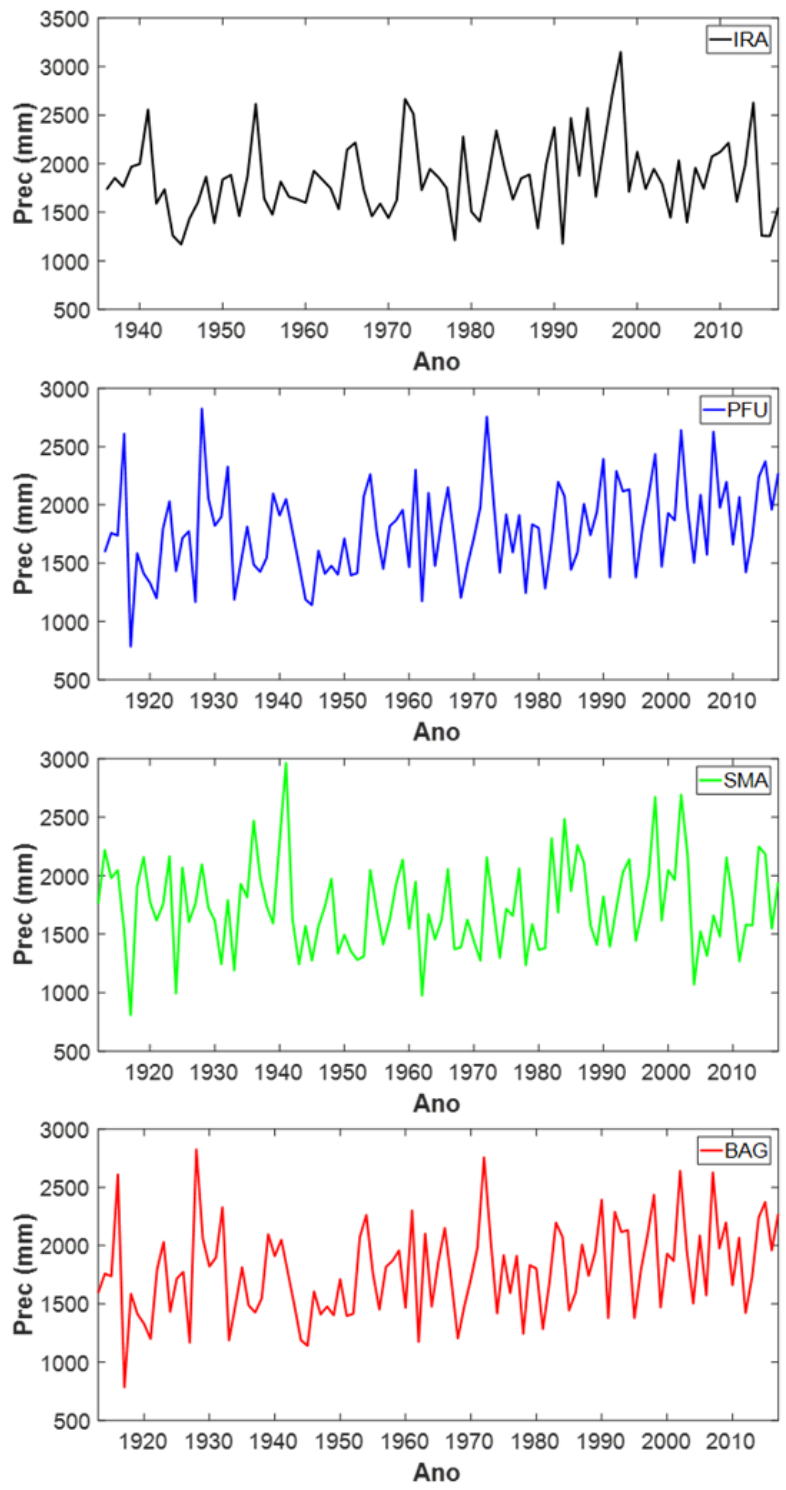

Figura 2 - Total de precipitação anual para as estações meteorológicas IRA, PFU, SMA e BAG.

O método ARIST, descrito em (Rigozo et al. 2005), consiste em ajustar, através de iterações sucessivas, os dados observados por uma função senoidal utilizando três parâmetros desconhecidos. Estes parâmetros são a amplitude, a frequência angular e a fase. O número máximo de iterações recomendado é de 200. O ARIST permite estimar o desvio padrão de cada um dos três parâmetros da função, possibilitando a determinação das periodicidades mais significativas considerando um nível de confiança de $95 \%$.

\section{Resultados}

De modo geral, as séries temporais dos totais anuais de precipitação mostram uma tendência de aumento nos valores de precipitação ao longo do tempo. Este aumento é mais visível nos dados as estações BAG e PFU. Os valores médios de total anual de precipitação detectados em cada estação foram de $\sim 1840 \mathrm{~mm}$ para IRA, $\sim 1780$ $\mathrm{mm}$ para PFU, $\sim 1730 \mathrm{~mm}$ para SMA e $\sim 1420 \mathrm{~mm}$ para BAG. Alguns valores baixos de precipitação são observados nos anos de 1945 ( 1100 mm), 1917 ( 780 $\mathrm{mm}), 1917(\sim 800 \mathrm{~mm})$ e $1917(\sim 750 \mathrm{~mm})$ nas estações IRA, PFU, SMA e BAG, respectivamente. Altos valores de precipitação são observados nos anos de 1998 ( 3000 mm), 1928 ( 2800 mm), 1941 ( 2900 mm) e $2002(\sim 2600 \mathrm{~mm})$ nas estações IRA, PFU, SMA e BAG, respectivamente. Os resultados obtidos através da aplicação da técnica ARIST estão apresentados na Figura 3.
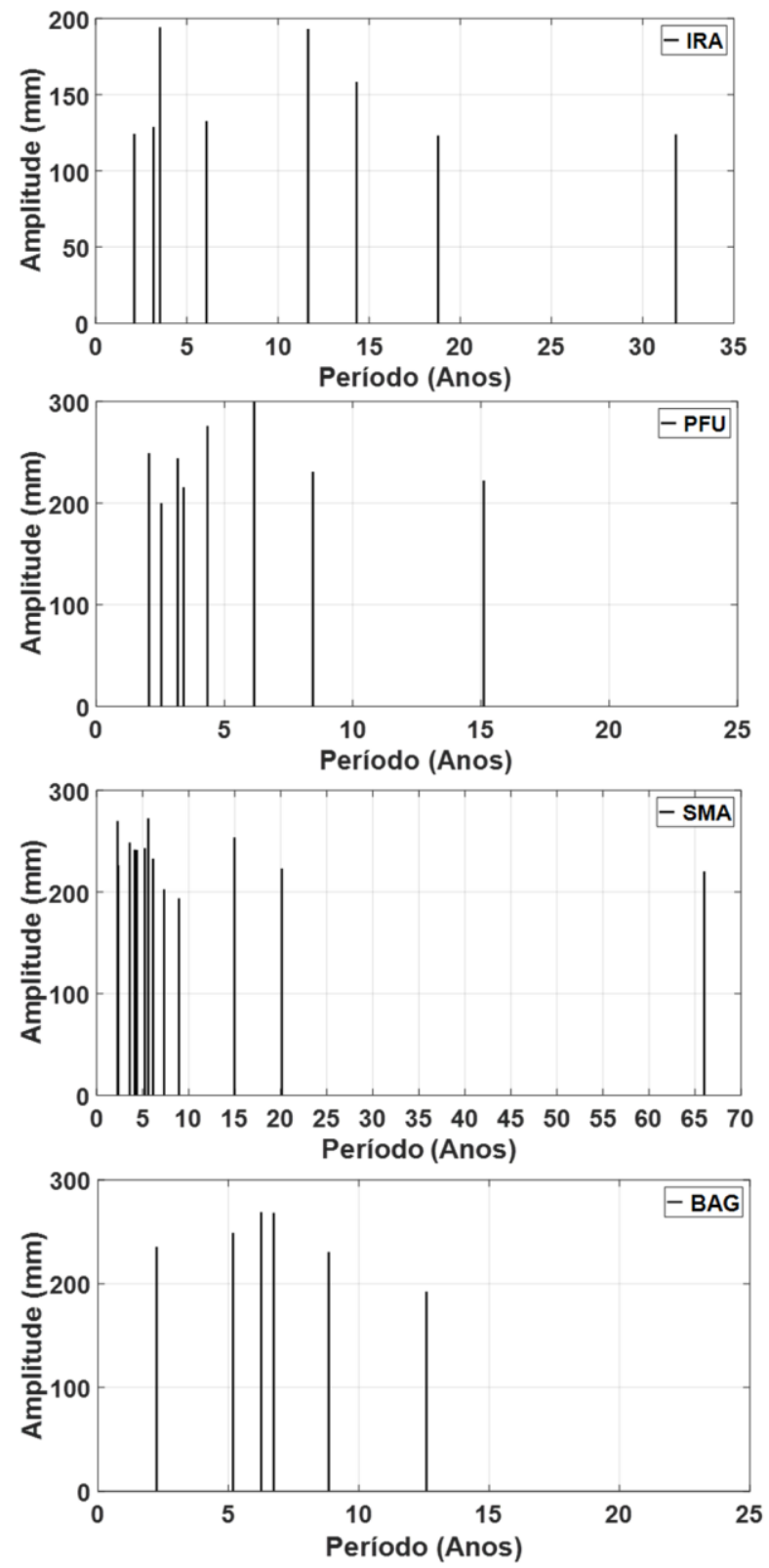

Figura 3 - Resultados estatisticamente significativos da ARIST, considerando o nível de 95\% de confiança, para os dados das estações IRA, PFU, SMA e BAG.

Periodicidades entre 2 a 7 anos foram detectadas em todas as séries temporais analisadas. Estas periodicidades são geralmente associadas ao fenômeno ENOS, que estão relacionados com alterações no padrão de temperatura na água do Oceano Pacífico. As amplitudes associadas as periodicidades entre 2 e 7 
anos, que chegam a valores próximos de $300 \mathrm{~mm}$, são as mais altas observadas nas quatro estações. Este resultado indica que o ENOS é o principal forçante, com característica periódica, que atuou sobre a variabilidade da precipitação pluviométrica no RS durante o último século.

Periodicidades de aproximadamente 11 anos, foram detectadas em todas as estações. Os valores de amplitudes associados a estas periodicidades chegaram a 200 mm para a estação IRA, $200 \mathrm{~mm}$ para a estação BAG, $250 \mathrm{~mm}$ para a estação PFU e $200 \mathrm{~mm}$ para a estação SMA. Esta faixa de periodicidades é geralmente associada ao ciclo solar de Schwabe ou, indiretamente, ao fluxo de raios cósmicos galácticos.

Variações periódicas com períodos de aproximadamente 22 anos foram observadas somente nas estações IRA e SMA, correspondendo a valores de amplitude de aproximadamente $125 \mathrm{~mm}$ e $220 \mathrm{~mm}$, respectivamente. Estes ciclos bidecadais são associados ao ciclo magnético solar de Hale ou, indiretamente, a variabilidade dos raios cósmicos galácticos (RCG). Além das periodicidades de $\sim 11$ e $\sim 22$ anos, foram identificadas periodicidades de 32 e 66 anos nos dados das estações IRA e SMA, respectivamente. Esta periodicidade pode ser associada a um harmônico do ciclo solar de Schwabe e ao ciclo solar de Gleissberg, respectivamente.

\section{Conclusão}

Os resultados deste trabalho indicam que os totais anuais de precipitação pluviométrica no RS apresentaram uma tendência de aumento durante o último século. São observados eventos marcantes de precipitação mais baixa e mais alta do que os valores médios. A análise espectral ARIST revelou a presença de periodicidades entre 2 e 7 anos e de $\sim 11$ anos em todas as estações meteorológicas investigadas. Periodicidades de aproximadamente $\sim 22$ anos foram detectadas em IRA e SMA. Para a estação SMA também foi identificada a periodicidade de $\sim 70$ anos.

As periodicidades detectadas, e correspondentes amplitudes, indicam que os principais forçantes periódicos que atuam sobre a precipitação pluviométrica no RS são o fenômeno ENOS e o ciclo solar de Schwabe. Nas estações de IRA e SMA, o ciclo solar de Hale também parece estar influenciando, de forma secundária, a variabilidade da precipitação. É interessante notar que os ciclos bidecadais, que podem estar relacionados a variabilidade dos RCG, são detectadas nas duas estações mais próximas a trajetória do centro da AMAS.

\section{Agradecimentos}

Os autores agradecem a Universidade Federal do Pampa (UNIPAMPA) pelo apoio institucional e ao INMET pela disponibilização dos dados meteorológicos utilizados. Matheus F. da Cruz e Giulia R. Herdies agradecem ao CNPq pela concessão de bolsa de Iniciação Científica PIBIC. Barbara B. Caprara agradece à UNIPAMPA pela concessão de bolsa de Iniciação Científica PDA. Everton Frigo agradece ao CNPq pela concessão de apoio financeiro (Edital Universal 01/2016, processo 429068/2016-6).

\section{Referências}

Echer, M.P.; Nordemann, D.J.R.; Rigozo, R. and Prestes, A. 2008. Wavelet analysis of a centennia (1895-1994) southern Brazil rainfall series (Pelotas, 3146'19"S, 52²0'33'’W). Clim. Change, 87, 489-497.

Frigo, E. 2013. Variações geomagnéticas, heliomagnéticas e do fluxo de raios cósmicos galácticos possíveis consequências climáticas na região da América do Sul. Tese de Doutorado - Universidade de São Paulo. 142p.

Rampelotto, P.H.; Rigozo, N.R.; Rosa, M.B.; Prestes, A.; Frigo, E.; Echer, M.P. and Nordemann, D.J.R. 2012. Variability of Rainfall and Temperature (1912-2008) from Santa Maria (29 $41^{\circ}$ 'S, $\left.53^{\circ} 48^{\prime} \mathrm{W}\right)$ and its Connection with Natural Influences. J. Atmos. Solar-Terr. Phys., 77, 152160.

Rigozo, N.R.; Echer, E.; Nordemann, D.J.R.; Luíz, E.A.V.; Heloisa, H.F. 2005. Comparative study between four classical spectral analysis methods, 411-430. 